\title{
Geologia
}

\section{Cálculo de Mapas de Probabilidade Diretamente dos Pesos da Krigagem O rdinária}

\author{
Calculation of Probability Maps Directly from 0 rdinary Kriging Weights \\ Jorge Kazuo Yamamoto (jkyamamo@usp.br) \\ Departamento de Geologia Sedimentar e Ambiental - Instituto de Geociências - USP \\ R. do Lago 562, CEP 05508-080, São Paulo, SP, BR
}

Recebido em 28 de abril de 2009; aceito em 10 de setembro de 2009

\section{RESUMO}

Mapas de probabilidade são úteis para interpretação de teores de minério ou grau de contaminação em solos, assim como para tomada de decisão em trabalhos exploratórios. Estes mapas de probabilidade são geralmente obtidos através da aplicação da krigagem das indicadoras. Os pesos da krigagem ordinária também podem ser usados para calcular mapas de probabilidade. Para testar os dois métodos, uma amostra foi retirada aleatoriamente de um conjunto completo. Deste conjunto completo, funções de distribuição acumulada foram determinadas. Assim, as funções de distribuição acumulada real e estimada foram comparadas. A grande maioria dos coeficientes de correlação entre os mapas de probabilidade estimados e reais é maior que 0,75 . O método da krigagem ordinária não somente funciona, mas também dá resultados ligeiramente melhores que a krigagem da indicadora da mediana. Além disso, os mapas de probabilidade calculados dos pesos da krigagem ordinária são muito mais fáceis que o método tradicional baseado tanto na krigagem da indicadora ou krigagem da indicadora da mediana.

Palavras-chave: Mapa de probabilidade; Krigagem da indicadora; Krigagem ordinária.

\begin{abstract}
Probability maps are useful to analyze ores or contaminants in soils and they are helpful to make a decision during exploration work. These probability maps are usually derived from the indicator kriging approach. Ordinary kriging weights can be used to derive probability maps as well. For testing these two approaches a sample data base was randomly drawn from an exhaustive data set. From the exhaustive data set actual cumulative distribution functions were determined. Thus, estimated and actual conditional cumulative distribution functions were compared. The vast majority of correlation coefficients between estimated and actual probability maps is greater than 0.75 . Not only does the ordinary kriging approach work, but it also gives slightly better results than median indicator kriging. Moreover, probability maps from ordinary kriging weights are much easier than the traditional approach based on either indicator kriging or median indicator kriging.
\end{abstract}

Keywords: Probability map; Indicator kriging; Ordinary kriging. 


\section{INTRODUÇÃO}

A técnica da krigagem da variável indicadora tem sido aplicada na geração de mapas de probabilidade. A variável aleatória é transformada em uma variável indicadora e esta é estimada em pontos não amostrados. Na realidade, a variável indicadora é obtida para cada teor de corte dentro dos limites da distribuição de frequências. Por exemplo, se a distribuição de frequências for dividida em 10 intervalos, então tem-se 9 decis. Para cada decil temse um valor correspondente de teor de corte que é utilizado para fazer a transformação não linear que resulta em 1 se a variável aleatória for menor ou igual ao teor de corte ou em zero se for maior. Para cada variável indicadora é calculado e modelado um variograma experimental. Geralmente, o número de teores de corte deve ser superior a 10 para que se garanta a representatividade da distribuição de frequências. Assim, este método requer um esforço muito grande na obtenção da variável indicadora, cálculo e modelagem de variogramas experimentais. Além disso, os variogramas das variáveis indicadoras, para teores de corte situados nas caudas da distribuição, dificilmente apresentam boa correlação espacial, haja vista a pequena porcentagem de pares possíveis. Os pares possíveis no cálculo de variogramas experimentais de variáveis indicadoras são somente aqueles pares que apresentam os valores 1 e zero, pois as outras combinações resultam em zero. Por exemplo, para o decil 0,10 , apenas $10 \%$ das indicadoras são iguais a 1 e no decil 0,9, apenas $10 \%$ são iguais a zero. Nesses casos, apenas $10 \%$ do total de pontos de dados podem formar pares que entram no cálculo da semivariância espacial. O melhor variograma experimental é obtido para a variável indicadora referente à mediana da distribuição, ou seja, o valor correspondente a 50\% da distribuição de frequências. Nesse caso, 50\% dos valores são iguais a 1 e $50 \%$ iguais a zero.

Outro problema associado à krigagem das indicadoras surge na construção da distribuição acumulada condicional, onde as indicadoras estimadas devem se apresentar monotônicas crescentes. Entretanto, isso nem sempre acontece, pois o uso de modelos de variogramas para diferentes teores de corte não garante que as indicadoras estimadas sejam sempre crescentes. Esse problema é conhecido na literatura como relação de ordem (Hohn, 1999). Com o objetivo de evitar esse tipo de problema, Deutsch e Journel (1992) propõem o uso da indicadora da mediana, em que somente um variograma experimental é calculado e modelado. Esse modelo é aplicado para a estimativa da indicadora para todos os teores de corte. Em termos rigorosos, essa aproximação não é bem aceita entre os geoestatísticos, mas é aquela que dá bons resultados e, evidentemente, não apresenta problemas de relação de ordem.
Uma alternativa à técnica da krigagem das indicadoras, para cálculo de mapas de probabilidade, foi proposta por Journel e Rao (1996), mas não tem sido muito utilizada, seja por desconhecimento, seja pela inexistência de programa de computador. A biblioteca GsLib de rotinas geoestatísticas (Deutsch e Journel, 1998) não disponibiliza esta opção de cálculo de mapas de probabilidade. A técnica proposta por Journel e Rao (1996) permite derivar a função de distribuição acumulada condicional diretamente dos pesos da krigagem ordinária. Essa técnica tem sido utilizada pelo autor devido à facilidade de implementação, bem como pela rapidez na obtenção de mapas de probabilidade. Esta opção está disponível nos programas Ordkrig2 e Ordkrig3 do Sistema GeoVisual (Yamamoto, 2001). Assim, para mostrar a viabilidade desta técnica, apresenta-se um estudo comparativo com a tradicional aproximação da krigagem das indicadoras no cálculo de mapas de probabilidade.

\section{REVISÃO DOS MÉTODOS CORRENTES}

Os métodos da krigagem das indicadoras, bem como a aproximação proposta por Journel e Rao (1996) serão apresentados neste item.

\section{Krigagem da indicadora da mediana}

A krigagem da indicadora é baseada em uma transformação não linear dos dados originais, que permite uma aproximação numérica da função de distribuição acumulada condicional (Olea, 1999). Dada uma função aleatória $Z(x)$, sua variável indicadora $I(x, z c)$ é obtida por uma transformação binária (Olea, 1999):

$$
I(x, z c)=\left\{\begin{array}{l}
0, \text { se } Z(x)>z c \\
1, \text { se } Z(x) \leq z c
\end{array}\right.
$$

onde zc é o teor de corte.

A média e variância de uma variável indicadora são:

$$
m=E[I(x, z c)]=\operatorname{Pr} o b[Z(x) \leq z c] ; \mathrm{e}
$$

$$
\begin{aligned}
\operatorname{Var}[I(x, z c)] & =E\left[I^{2}(x, z c)\right]-(E[I(x, z c)])^{2} \\
& =m-m^{2}=\operatorname{Pr} o b[Z(x) \leq z c](1-\operatorname{Pr} o b[Z(x) \leq z c])
\end{aligned}
$$

O valor máximo da média é 0,5 para um teor de corte correspondente à mediana da distribuição. Portanto, a variância máxima é 0,25 . Observe-se que para qualquer média menor ou maior que 0,5 a variância é menor que 0,25 . Na realidade, a variância da variável indicadora em função da média é descrita por uma função quadrática (Figura 1). 
A krigagem da indicadora da mediana é baseada em um modelo de semivariograma que alcança a variância máxima da variável indicadora. A variável indicadora pode ser estimada em um ponto não amostrado $\left(x_{o}\right)$ como:

$$
i_{K O}^{*}\left(x_{o}, z c\right)=\sum_{j=1}^{n} \lambda_{j} i\left(x_{j}, z c\right)
$$

onde $i_{K O}^{*}\left(x_{o}, z c\right)$ é a estimativa por krigagem ordinária da variável indicadora; $i\left(x_{j}, z c\right)$ é o valor da indicadora para a j-ésima amostra e um teor de corte $z c$ e $\lambda_{j}$ é o peso da krigagem ordinária para a j-ésima amostra.

Como a krigagem da indicadora da mediana é baseada no semivariograma da indicadora da mediana, o conjunto de pesos da krigagem é o mesmo para todos os teores de corte.

\section{Krigagem ordinária}

A estimativa pela krigagem ordinária $z_{K O}^{*}\left(x_{o}\right)$ em um ponto não amostrado $\left(x_{o}\right)$ é obtida como uma combinação linear dos vizinhos próximos:

$$
z_{K O}^{*}\left(x_{o}\right)=\sum_{i=1}^{n} \lambda_{i} z\left(x_{i}\right)
$$

onde $z_{K O}^{*}\left(x_{o}\right)$ é o valor estimado no ponto $\left(x_{o}\right) ; z\left(x_{i}\right)$ é o valor da variável aleatória no i-ésimo ponto e $\lambda_{i}$ é o peso correspondente calculado pela krigagem ordinária.

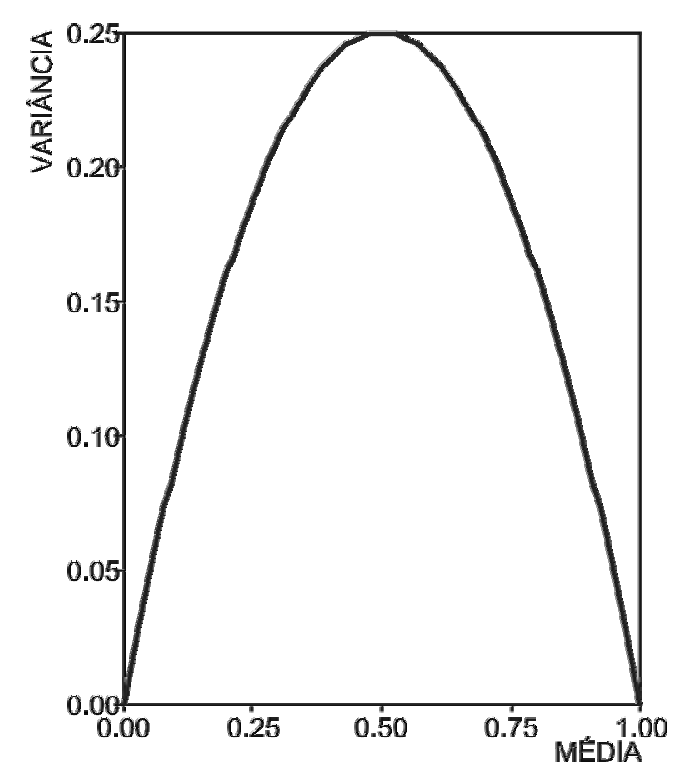

Figura 1. Função quadrática que descreve a variância da variável indicadora em função da média.
Journel e Rao (1996) interpretaram os pesos da krigagem ordinária como probabilidade condicional $\left\{\lambda_{i}, i=1, n\right\}$. Essa interpretação só é possível se todos os pesos da krigagem ordinária forem positivos e com soma igual a 1. Portanto, isso significa que se um peso negativo for encontrado, este deve ser substituído. O algoritmo para substituição de pesos negativos, proposto por Journel e Rao (1996), consiste em adicionar uma constante, igual ao módulo do maior peso negativo, a todos os pesos. Em seguida, os pesos são normalizados novamente para soma igual a 1 :

$$
\tau_{i}=\frac{\lambda_{i}+C}{\sum_{i=1}^{n}\left(\lambda_{i}+C\right)} \text { para } i=1, n
$$

onde $C=\left|\lambda_{j}\right|$ é o maior peso negativo em módulo. Assim, o algoritmo elimina apenas a amostra com o maior peso negativo em módulo e, caso exista, mais amostras com pesos negativos, os seus pesos serão substituídos pela adição da constante $C$.

A estimativa da krigagem ordinária, após a correção dos pesos negativos, no ponto não amostrado $\left(x_{o}\right)$, torna-se:

$$
z_{K O}^{*}\left(x_{o}\right)=\sum_{i=1}^{n} \tau_{i} z\left(x_{i}\right)
$$

Após a aplicação do algoritmo para eliminação de pesos negativos, os pesos corrigidos da krigagem ordinária satisfazem as condições para que possam ser interpretados como probabilidades condicionais. Assim, pode-se formar a função de distribuição acumulada condicional em uma dada localização $\left(x_{o}\right)$, classificando os $n$ valores vizinhos da variável aleatória em ordem crescente:

$$
z\left(x_{1}\right) \leq z\left(x_{2}\right) \leq \ldots \leq z\left(x_{n}\right)
$$

A função de distribuição acumulada condicional no ponto $\left(x_{o}\right)$ pode ser calculada como:

$$
F\left(x_{o}, z_{\alpha}\right)=\sum_{i=1}^{\alpha} \tau_{\alpha}
$$

Para ilustrar o procedimento, considere-se o conjunto de valores e pesos apresentado na Tabela 1.

Os valores e pesos correspondentes são classificados em ordem crescente e os pesos acumulados, conforme a Tabela 2.

A função de distribuição acumulada assim obtida pode ser representada graficamente (Figura 2).

A partir da Figura 2, pode-se determinar a probabilidade para qualquer valor no intervalo [1,3 - 10,0]. Por exemplo, a probabilidade da variável aleatória ser menor que 
3,5 é igual a 0,30; e a probabilidade da variável aleatória ser menor que 5,0 é igual a 0,467.

Observe-se que para essa função de distribuição acumulada condicional, derivada dos pesos da krigagem ordinária, a média condicional é a própria estimativa da kri-

Tabela 1 . Valores da variável aleatória no ponto $\left(\mathrm{x}_{0}\right)$ e respectivos pesos da krigagem ordinária.

\begin{tabular}{cc}
\hline Valores & Pesos \\
\hline 8,3 & 0,18 \\
3,5 & 0,12 \\
6,0 & 0,10 \\
2,2 & 0,13 \\
4,8 & 0,15 \\
7,0 & 0,15 \\
1,3 & 0,05 \\
10,0 & 0,12 \\
\hline
\end{tabular}

Tabela 2. O btenção da função de distribuição acumulada condicional no ponto $\left(x_{0}\right)$.

\begin{tabular}{ccc}
\hline Valores & Pesos & FDAC \\
\hline 1,3 & 0,05 & 0,05 \\
2,2 & 0,13 & 0,18 \\
3,5 & 0,12 & 0,30 \\
4,8 & 0,15 & 0,45 \\
6,0 & 0,10 & 0,55 \\
7,0 & 0,15 & 0,70 \\
8,3 & 0,18 & 0,88 \\
10,0 & 0,12 & 1,00 \\
\hline
\end{tabular}

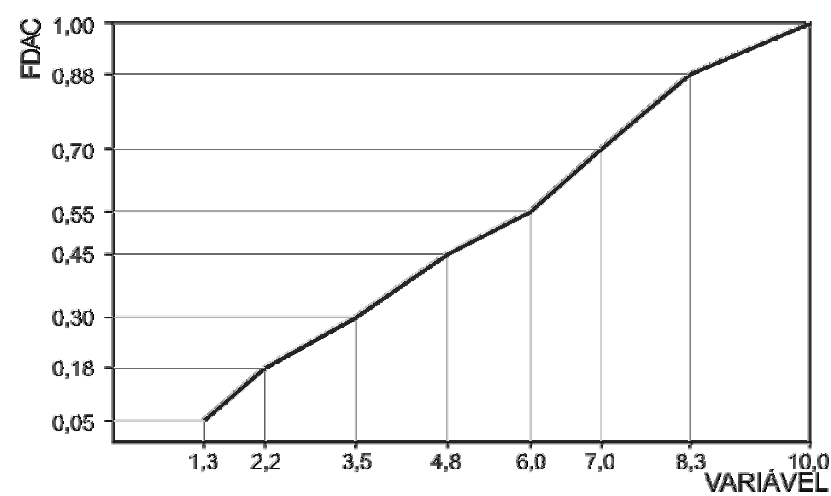

Figura 2. Função de distribuição acumulada condicional construída com os dados da Tabela 2. gagem ordinária (equação 2) e a variância condicional é a variância de interpolação (Yamamoto, 2000):

$$
s_{o}^{2}=\sum_{i=1}^{n} \tau_{i}\left[z\left(x_{i}\right)-z\left(x_{o}\right)\right]^{2}
$$

Na verdade, Journel e Rao (1996) proporcionaram a fundamentação teórica para a variância de interpolação, ou seja, a variância condicional associada à função de distribuição acumulada condicional construída a partir dos pesos da krigagem ordinária. A variância de interpolação foi proposta originalmente por Yamamoto (1991), como uma aproximação empírica, mas funcional. Assim, a sua publicação em um periódico internacional foi viabilizada com a interpretação dos pesos da krigagem ordinária como probabilidades condicionais associadas aos valores da vizinhança.

\section{MATERIAIS E MÉTODOS}

Para o estudo comparativo que se pretende desenvolver neste artigo, uma amostra aleatória estratificada com 144 pontos foi extraída de um conjunto completo com 2500 pontos de dados distribuídos em uma malha regular de $50 \mathrm{x}$ 50 pontos. A Figura 3 ilustra a variação espacial do conjunto completo e a amostra aleatória estratificada dela extraída. O conjunto completo poderia representar teores de ouro (ppm) em amostras de solo, numa área de 50 por $50 \mathrm{~m}$, com amostras coletadas em uma malha regular de $1 \mathrm{~m}$. Devido ao caráter sigiloso de campanhas de prospecção, bem como de investigação de uma área degradada, tais informações dificilmente são cedidas para pesquisa. Assim, a solução é empregar dados sintéticos que simulem situações reais, as quais podem ser utilizadas para desenvolvimento e teste de novos algoritmos computacionais.

Para validar a amostra quanto à sua representatividade, deve-se comparar as distribuições de frequências (Figura 4).

Como se pode observar na Figura 4, há uma boa aderência entre as frequências acumuladas do conjunto completo e da amostra estratificada. A distância média (Yamamoto, 2005) medida no diagrama P-P é de apenas 1,09. Portanto, a amostra extraída pode ser considerada representativa da população. A Tabela 3 apresenta os parâmetros da população e as estatísticas amostrais.

Pode-se verificar nesta tabela que o desvio padrão amostral é menor que o populacional, o que explica o menor coeficiente de variação. Evidentemente, se o processo de amostragem aleatória estratificada fosse feito interativamente, seria possível chegar a uma amostra com as estatísticas mais próximas dos parâmetros populacionais. Mas, deve-se ressaltar que nunca se conhece a população e, portanto, tem-se que extrair da amostra as estatísticas que serão utilizadas para inferir os parâmetros populacionais. 

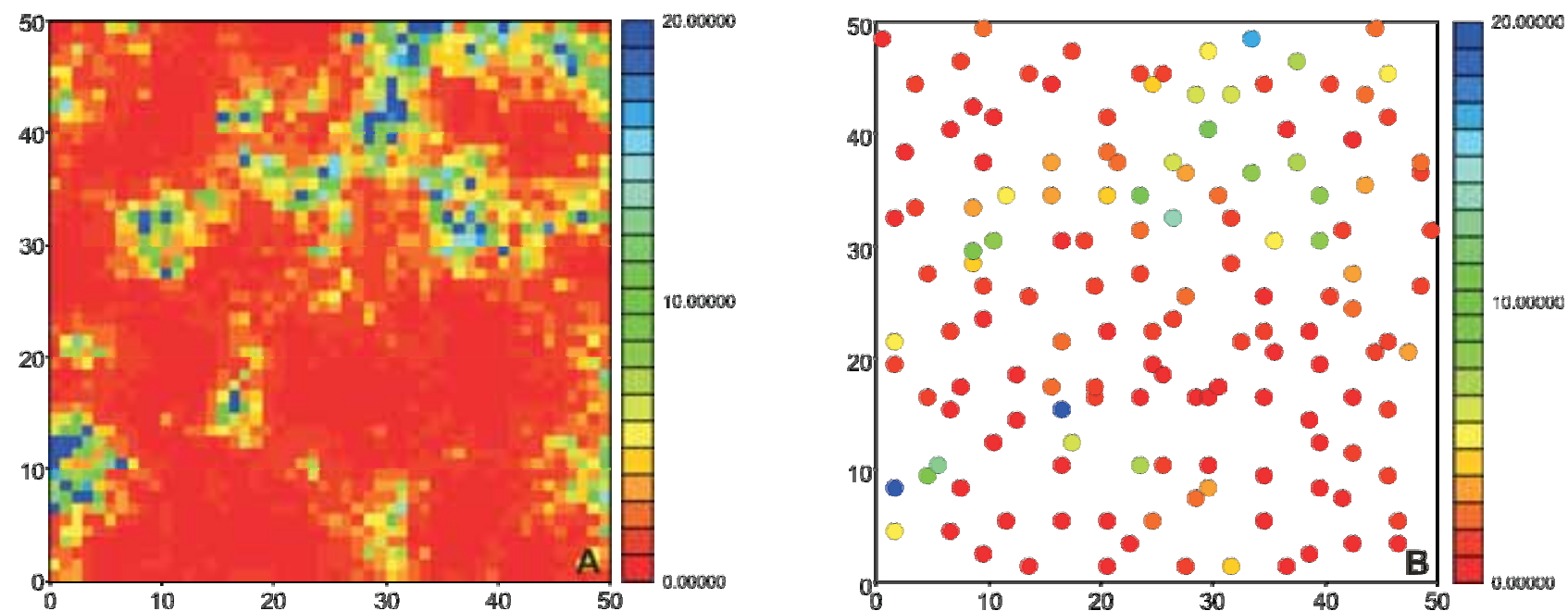

Figura 3. Imagem do conjunto completo (A) e o mapa de localização da amostra aleatória estratificada (B).

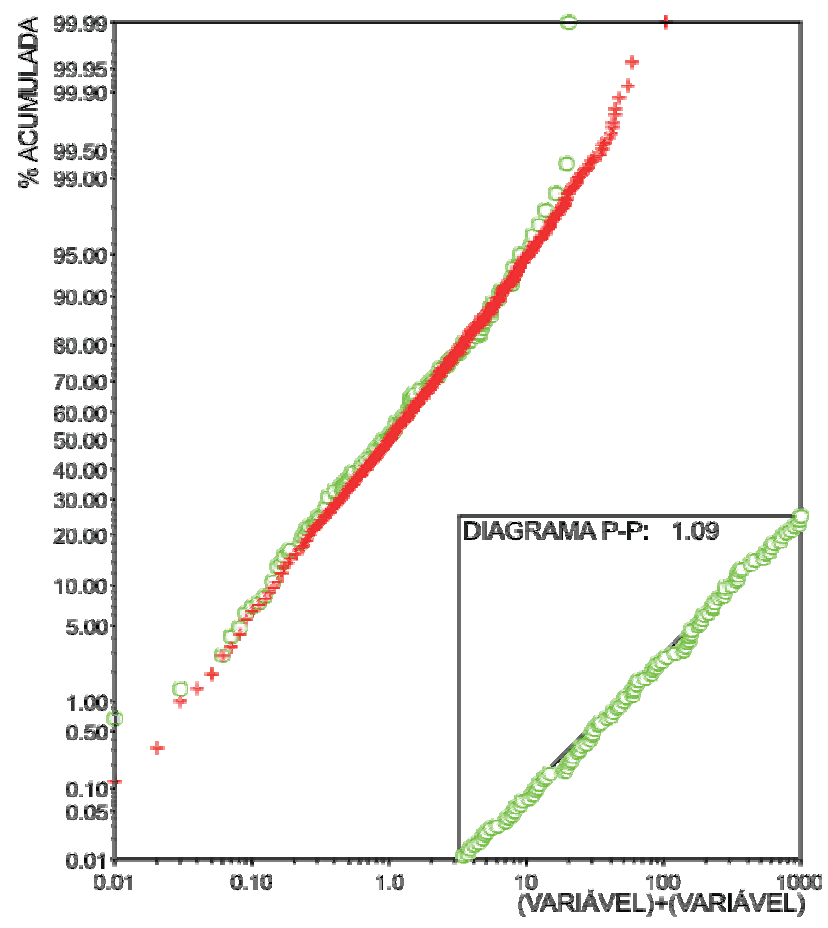

Figura 4. Distribuições de frequências acumuladas e diagrama P-P. Legenda: Cruz vermelha = conjunto completo; círculo verde = amostra extraída. No diagrama P-P: eixo das abscissas representa a probabilidade calculada; eixo das ordenadas representa a probabilidade de referência.

Para o caso em estudo, foram calculados variogramas omnidirecionais, haja vista o fenômeno em estudo ser isotrópico (Figura 5).

A amostra com a variável original e a sua transformada para indicadora da mediana constituem os materiais para o presente estudo.
Tabela 3. Parâmetros da população e estatísticas da amostra de estudo.

\begin{tabular}{ccc}
\hline & Parâmetros & Estatísticas \\
\hline NN$^{\circ}$ de dados & 2500 & 144 \\
Média & 2,580 & 2,337 \\
Desvio padrão & 5,151 & 3,564 \\
Coef. de variação & 1,996 & 1,525 \\
Máximo & 102,700 & 20,350 \\
Quartil superior & 2,555 & 2,680 \\
Mediana & 0,959 & 0,890 \\
Quartil inferior & 0,333 & 0,310 \\
Mínimo & 0,010 & 0,010 \\
\hline
\end{tabular}

Os mapas de probabilidade serão calculados com base na krigagem da indicadora da mediana, bem como diretamente dos pesos da krigagem ordinária. Os mapas de probabilidade serão calculados somente dentro do domínio da fronteira convexa dos pontos amostrais, conforme algoritmo proposto por Yamamoto (1997).

Como no caso em estudo existe um conjunto completo, do qual foi extraída a amostra aleatória estratificada, é possível calcular as funções de distribuição acumulada reais. Essas funções podem ser usadas para conferir a validade dos pesos da krigagem ordinária para derivar os mapas de probabilidade, conforme proposta de Journel e Rao (1996). Assim, dado um conjunto completo, pode-se construir uma função de distribuição acumulada real usando os dados da vizinhança em relação ao ponto central. Por exemplo, para qualquer ponto central (exceto nas bordas da malha regular) há 8 pontos vizinhos (Figura 6A). Expandindo a vizinhança tem-se mais 16 pontos, totalizando 24 pontos (Figura 6B) e assim por diante. 

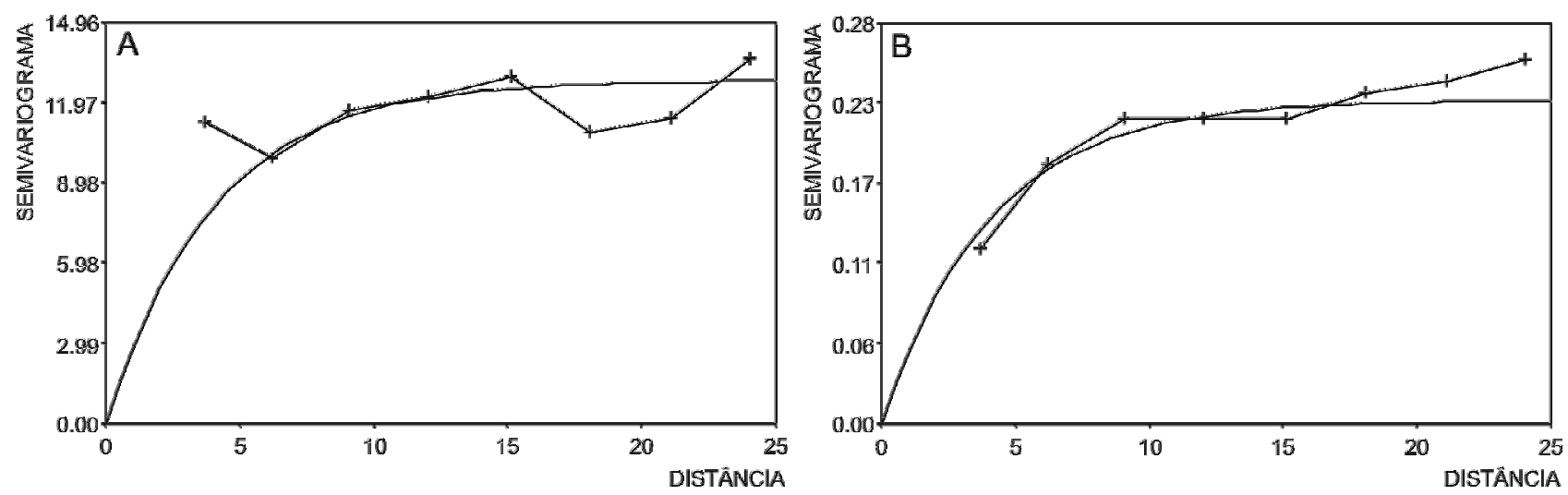

Figura 5. Semivariogramas omnidirecionais para os dados originais (A) e para a variável indicadora da mediana (B).

Para determinar as funções de distribuição acumulada reais, foi considerada uma vizinhança de 24 pontos. Todos os dados vizinhos mais o dado do ponto central são classificados em ordem crescente para obtenção da função de distribuição acumulada real. Observe-se que cada ponto pode contribuir com um peso constante (1/25) ou variável conforme o inverso da distância, sendo 0,1111 para o ponto central; 0,0555 para cada um dos 8 pontos da primeira auréola e 0,0277 para cada um dos 16 pontos da segunda auréola. A Figura 7 ilustra o procedimento de atribuição de probabilidades constantes ou variáveis conforme o inverso da distância.

Exemplos de cálculos da probabilidade real são apresentados nas Figuras 8 e 9.

As probabilidades constantes serão denominadas doravante PPC e as variáveis conforme o inverso da distância PPV. As funções de distribuição acumuladas condicionais a partir da krigagem da indicadora da mediana serão denominadas PKI e PKO as mesmas derivadas diretamente dos pesos da krigagem ordinária.
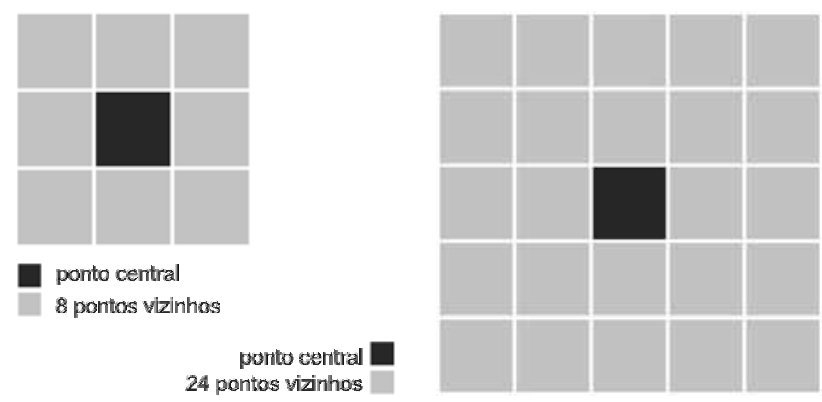

Figura 6. Vizinhanças em torno do ponto central. 8 vizinhos próximos (A) e 24 vizinhos próximos (B).

\section{RESULTADOS E DISCUSSÃO}

Antes de se proceder aos cálculos propriamente ditos, deve-se decidir o número de teores de corte. Nesse caso, a distribuição de frequências amostral foi dividida em 20 intervalos, ou seja, 19 teores de corte $(0,081 ; 0,136 ; 0,168$; 0,$234 ; 0,310 ; 0,348 ; 0,464 ; 0,616 ; 0,740 ; 0,890 ; 1,091$; $1,364 ; 1,460 ; 2,016 ; 2,680 ; 3,414 ; 4,728 ; 6,250 ; 9,004)$, equivalentes aos percentis de 0,05 a 0,95 em intervalos de 0,05 . Portanto, a função de distribuição acumulada condicional será construída com base em 19 pontos, tanto para a krigagem da indicadora da mediana como para aquela derivada diretamente dos pesos da krigagem ordinária.

Com o objetivo de ilustrar os procedimentos adotados, a Figura 10 apresenta as funções de distribuição acumulada estimadas e reais para dois pontos $(31,50 ; 3,50)$ e $(16,50 ; 25,50)$.
PESO CONSTANTE

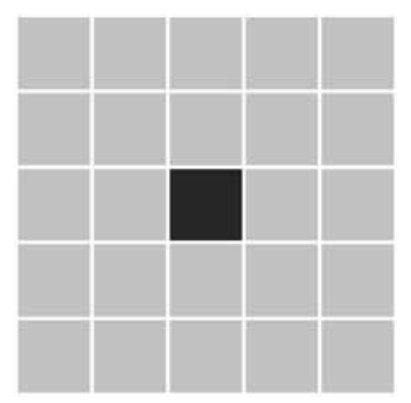

ponto central

peso $=0.04$
PESO VARIÁVEL

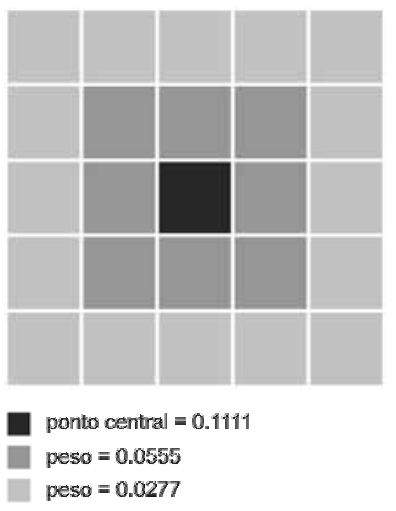

Figura 7. Desenho mostrando o processo de atribuição de probabilidades constantes $(A)$ e variáveis conforme o inverso da distância (B). 

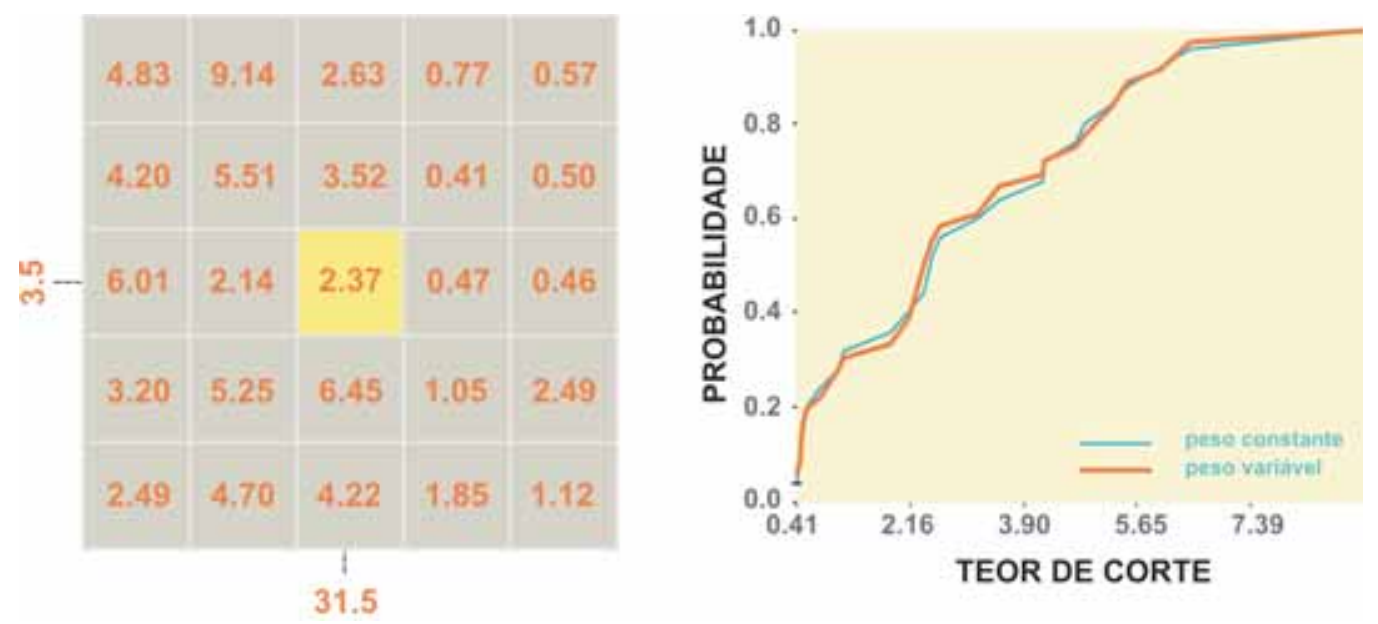

Figura 8. Cálculo das probabilidades reais para o ponto $(31,5 ; 3,5)$ com base no peso constante e peso variável.
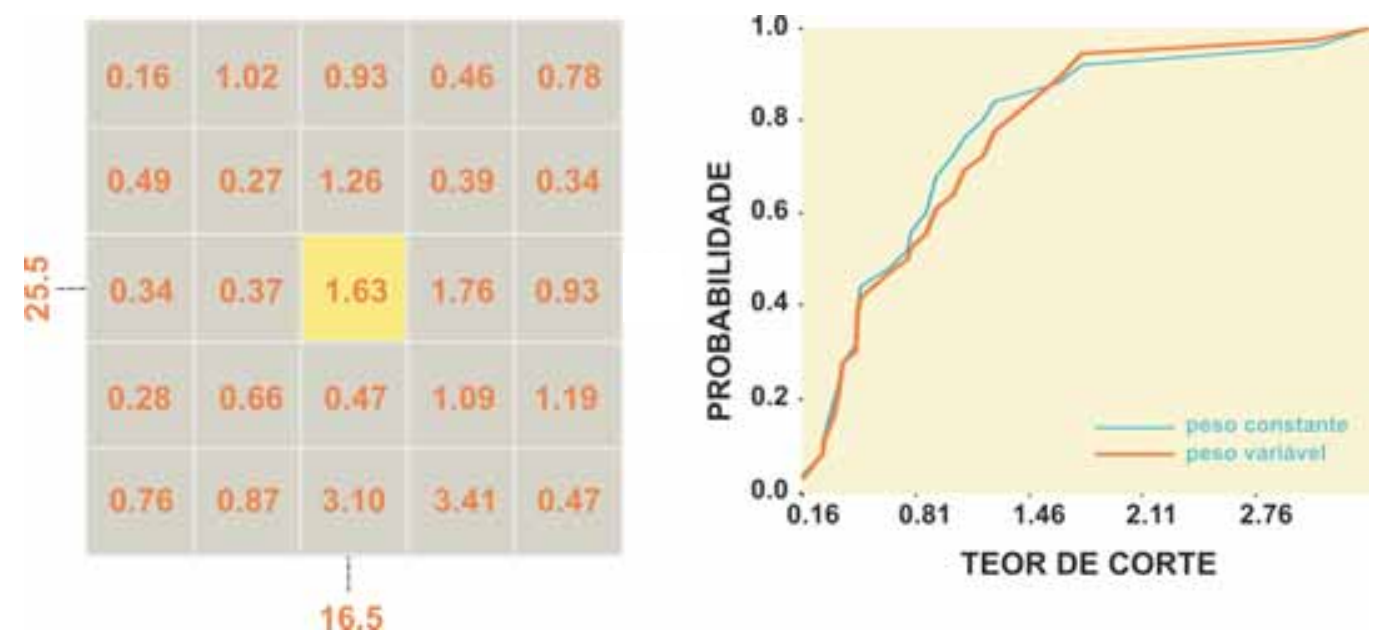

Figura 9. Cálculo das probabilidades reais para o ponto $(16,5 ; 25,5)$ com base no peso constante e peso variável.
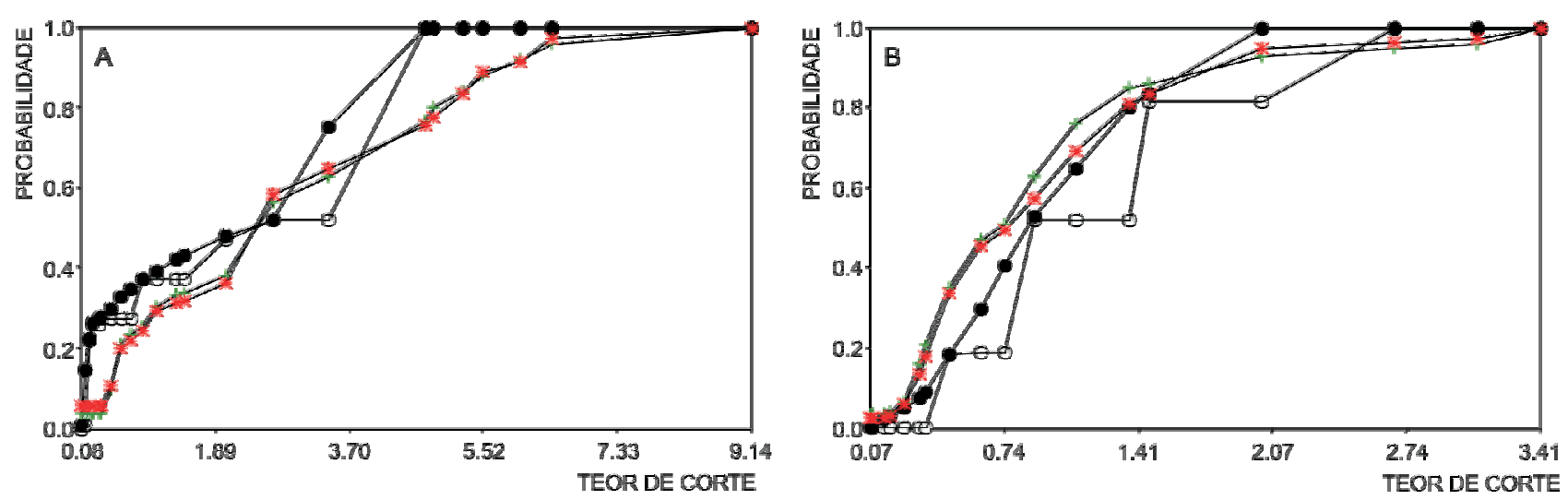

Figura 10. Funções de distribuição acumulada para o ponto $(31,50 ; 3,50)(A)$ e para o ponto $(16,50 ; 25,50)(B)$. Legenda: círculo vazio $=$ PKI; círculo cheio $=$ PKO ; mais $=$ PPC e asterisco $=$ PPV. 
Como se pode observar nesta figura, a principal diferença entre as funções geradas pela krigagem da indicadora da mediana e pelos da krigagem ordinária é que a primeira é monotônica crescente, mas em degraus, enquanto a segunda é mais contínua.

A Tabela 4 lista os coeficientes de correlação calculados para as funções estimadas e reais.

Como se pode observar, a PKO apresenta maiores correlações que a PKI. Isso significa que a função de distribuição acumulada estimada pela krigagem ordinária é a que melhor se ajusta à função de distribuição acumulada real. O procedimento de cálculo de coeficientes de corre- lação entre os diferentes métodos é repetido para todos os nós estimados. Os resultados são apresentados na forma de histogramas para as diferentes correlações (Figura 11).

Nesta figura, é possível observar que a grande maioria das correlações é maior que 0,90 (65,9\% para PKIxPPC; 63,9\% para PKIxPPV; 75,1\% para PKOxPPC e 72,8\% para PKOxPPV). A krigagem ordinária proporciona correlações melhores que a própria krigagem da indicadora da mediana. Além disso, a função de distribuição acumulada real obtida com peso constante é a que melhor se correlaciona com a função de distribuição acumulada condicional derivada dos pesos da krigagem ordinária.

Tabela 4. Coeficientes de correlação para as funções de distribuição acumulada nos pontos $(31,50 ; 3,50)$ e $(16,50 ; 25,50)$.

\begin{tabular}{ccccc}
\hline Ponto & PKI x PPC & PKI x PPV & PKO x PPC & 0,97285 \\
$(31,50 ; 3,50)$ & 0,95964 & 0,95669 & 0,97077 \\
$(16,50 ; 25,50)$ & 0,95408 & 0,96231 & 0,98647 & 0,99092 \\
\hline
\end{tabular}
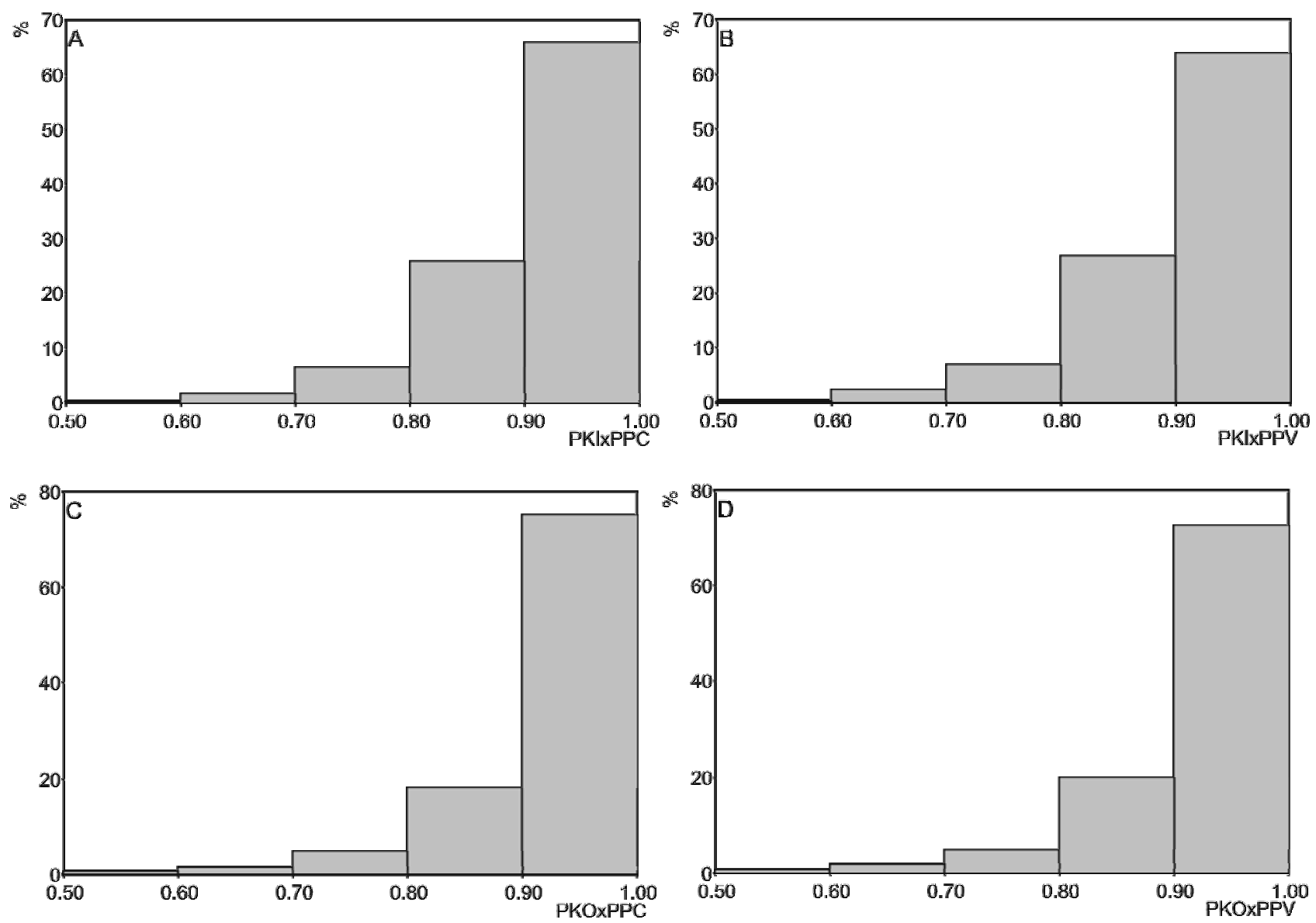

Figura 11. Histogramas de coeficientes de correlação entre as funções de distribuição acumuladas reais e estimadas: PKI x PPC (A); PKI x PPV (B); PKO x PPC (C); PKO x PPV (D). 
Agora, pode-se comparar os mapas de probabilidade calculados conforme PKI e PKO. Os mapas de probabilidade foram calculados para os 19 percentis sobre a curva de distribuição acumulada. Os coeficientes de correlação entre os mapas de probabilidade estimados e reais encontram-se listados na Tabela 5 e apresentados graficamente na Figura 12.

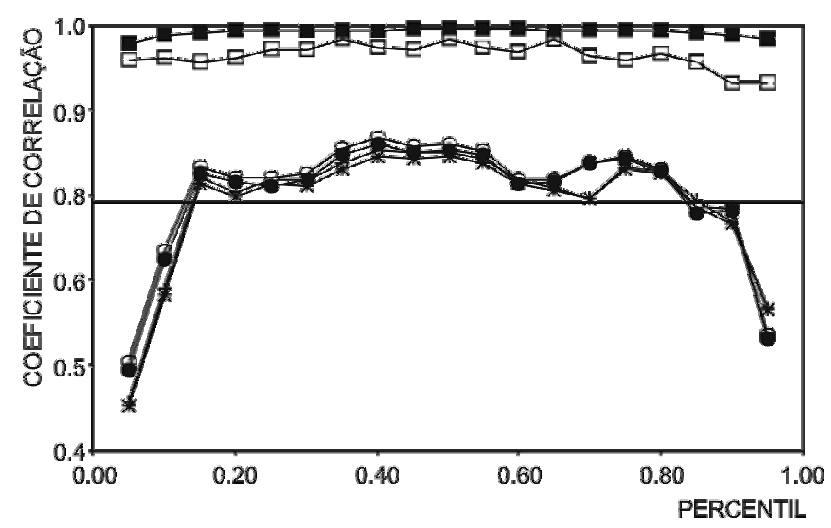

Figura 12. Coeficientes de correlação medidos entre mapas de probabilidade nos 19 percentis. Legenda: mais $=$ PKI $\times$ PPC; asterisco = PKI $\times$ PPV; círculo vazio $=$ PKO $\times$ PPC; círculo cheio $=$ PKO $\times$ PPV; quadrado vazio $=$ PKI $\times$ PKO ; quadrado cheio $=$ PPC $\times$ PPV.
Conforme os valores apresentados na Tabela 5 e Figura 12 , pode-se verificar que as probabilidades reais calculadas pelo peso constante apresentam-se mais correlacionadas às probabilidades estimadas tanto pela PKI como pela PKO. Entre os métodos de estimativa de probabilidades, verifica-se que as probabilidades estimadas pela PKO se aproximam mais às probabilidades reais. Na verdade, dos 19 teores de corte, 16 apresentam maiores valores que a PKI. As exceções são verificadas nos percentis 0,600, 0,850 e 0,950. As correlações máximas são verificadas para o teor de corte equivalente à mediana da distribuição amostral. As correlações entre as probabilidades estimadas pela PKI e PKO são altas em torno da mediana, mas caem muito na cauda superior da distribuição. Em relação às probabilidades reais, as correlações são sempre altas, mas também diminuem nas caudas da distribuição. A partir dos dados apresentados, pode-se concluir que os mapas de probabilidade calculados diretamente dos pesos da krigagem ordinária são confiáveis e se aproximam mais aos mapas de probabilidade reais. Portanto, a alternativa apresentada por Journel e Rao (1996) é viável e apresenta a vantagem de poder ser obtida durante o processo de estimativa por krigagem ordinária.

As Figuras 13, 14 e 15 apresentam os mapas de probabilidade reais e estimados, respectivamente para o quartil inferior, mediana e quartil superior.

Tabela 5. Coeficientes de correlação entre mapas de probabilidade estimados e reais.

\begin{tabular}{cccccccc}
\hline Percentil & Teor de corte & PKI x PPC & PKI x PPV & PKO x PPC & PKO x PPV & PKI x PKO & PPC x PPV \\
\hline 0,05 & 0,081 & 0,47086 & 0,46304 & 0,52719 & 0,51509 & 0,95164 & 0,97487 \\
0,10 & 0,136 & 0,63019 & 0,62006 & 0,68333 & 0,66982 & 0,95316 & 0,98607 \\
0,15 & 0,168 & 0,78569 & 0,77726 & 0,80204 & 0,79075 & 0,94989 & 0,98954 \\
0,20 & 0,234 & 0,76655 & 0,75914 & 0,78744 & 0,77909 & 0,95324 & 0,99169 \\
0,25 & 0,31 & 0,78254 & 0,77662 & 0,78503 & 0,77560 & 0,96615 & 0,99241 \\
0,30 & 0,348 & 0,78310 & 0,77475 & 0,79270 & 0,78281 & 0,96491 & 0,99289 \\
0,35 & 0,464 & 0,80562 & 0,76991 & 0,82772 & 0,81841 & 0,98021 & 0,99345 \\
0,40 & 0,616 & 0,82367 & 0,81612 & 0,84185 & 0,83313 & 0,96851 & 0,99416 \\
0,45 & 0,74 & 0,82107 & 0,81231 & 0,83019 & 0,82114 & 0,96580 & 0,99450 \\
0,50 & 0,89 & 0,82270 & 0,81449 & 0,83296 & 0,82487 & 0,98197 & 0,99481 \\
0,55 & 1,091 & 0,81324 & 0,80743 & 0,82554 & 0,81895 & 0,97061 & 0,99475 \\
0,60 & 1,364 & 0,78421 & 0,77806 & 0,78206 & 0,77677 & 0,96263 & 0,99443 \\
0,65 & 1,460 & 0,77266 & 0,76983 & 0,78272 & 0,78032 & 0,98206 & 0,99435 \\
0,70 & 2,016 & 0,75769 & 0,75753 & 0,80656 & 0,80542 & 0,95715 & 0,99363 \\
0,75 & 2,680 & 0,80126 & 0,79793 & 0,81588 & 0,81188 & 0,95221 & 0,99273 \\
0,80 & 3,414 & 0,79447 & 0,79092 & 0,79878 & 0,79365 & 0,96013 & 0,99235 \\
0,85 & 4,728 & 0,75348 & 0,74339 & 0,74346 & 0,73531 & 0,94924 & 0,98975 \\
0,90 & 6,250 & 0,72515 & 0,72073 & 0,74312 & 0,73897 & 0,91916 & 0,98706 \\
0,95 & 9,004 & 0,60227 & 0,60008 & 0,56437 & 0,56014 & 0,91890 & 0,98161 \\
\hline
\end{tabular}



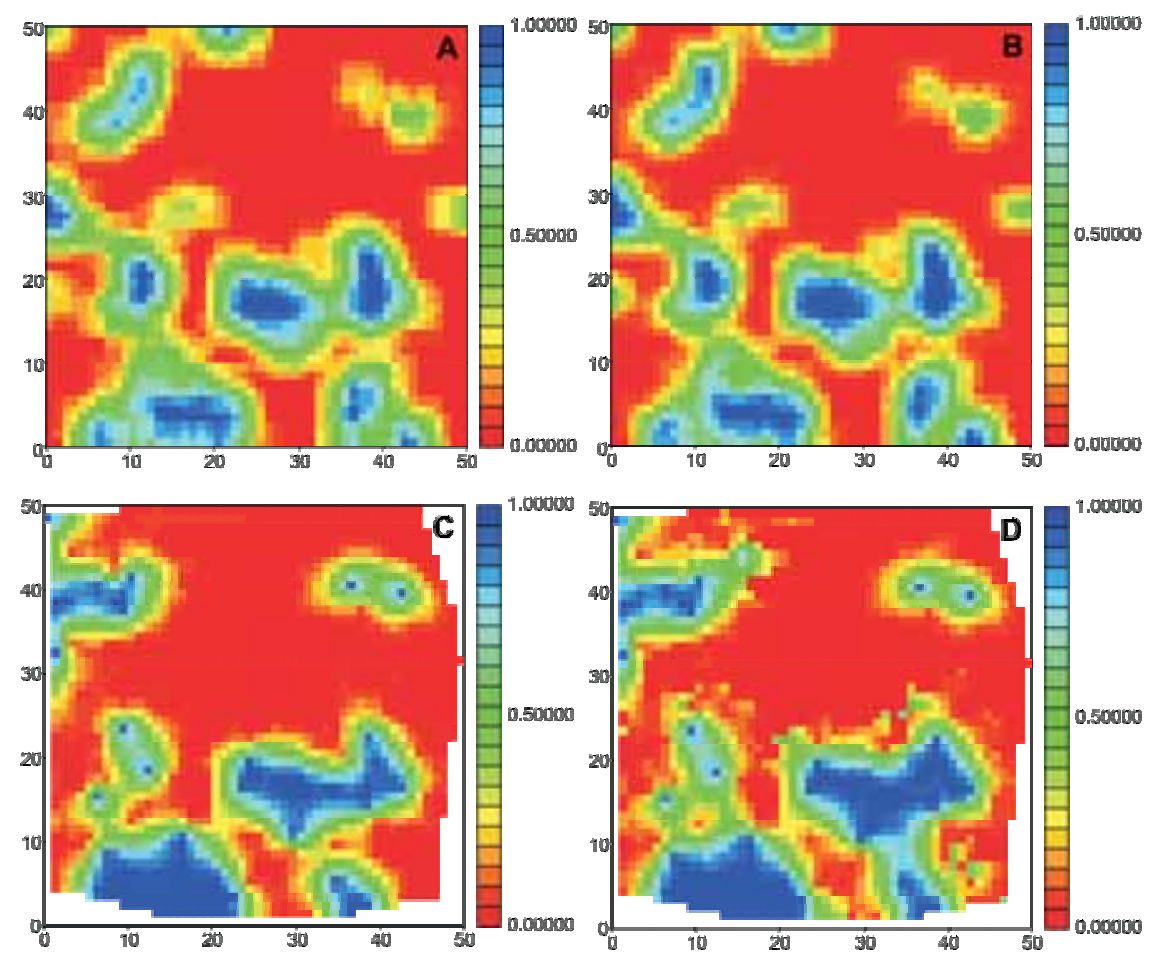

Figura 13. Mapas de probabilidades reais: peso constante (A) e peso variável (B) e mapas de probabilidade estimados pela PKI (C) e pela PKO (D) (teor de corte $=0,310$ ).
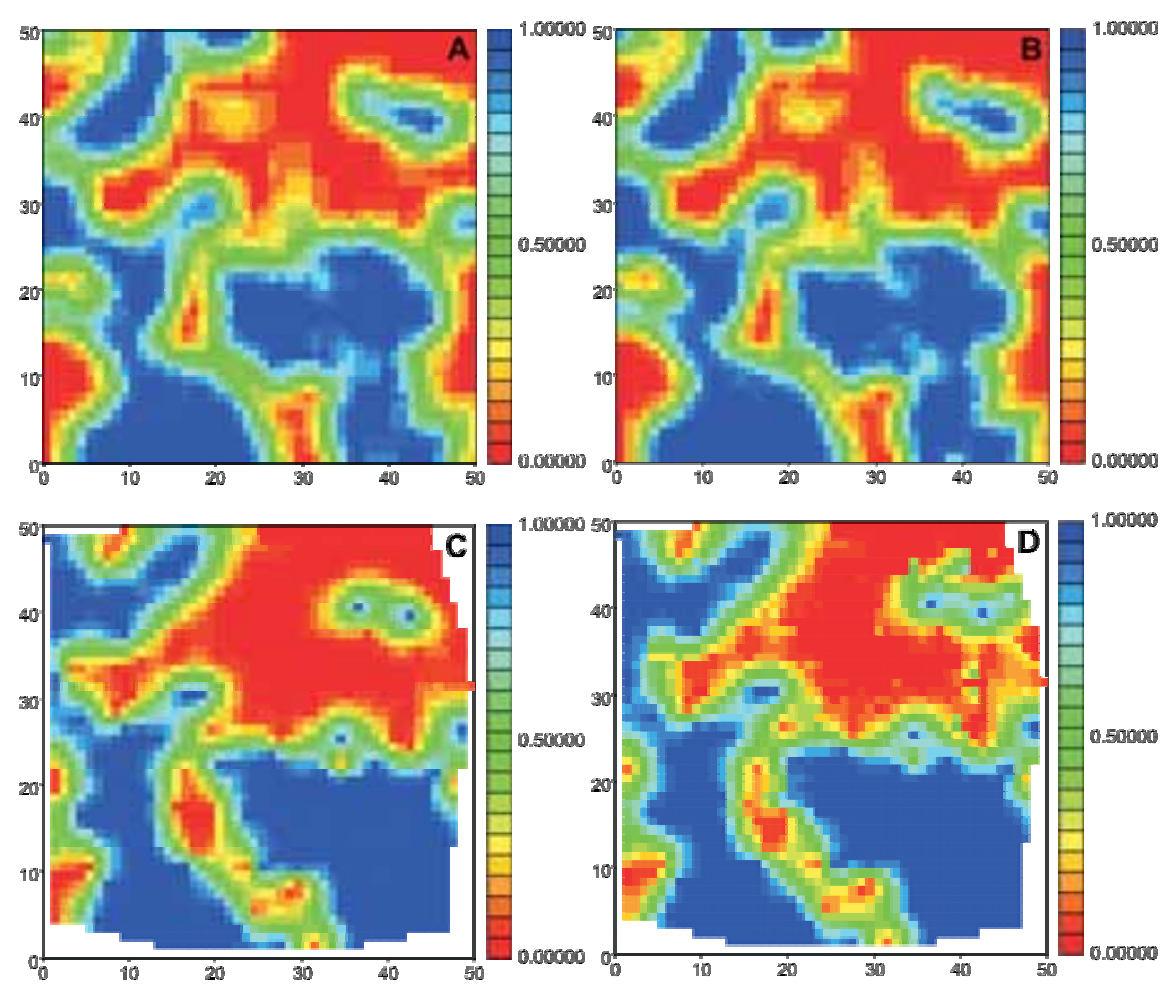

Figura 14. Mapas de probabilidades reais: peso constante (A) e peso variável (B) e mapas de probabilidade estimados pela PKI (C) e pela PKO (D) (teor de corte $=0,890$ ). 

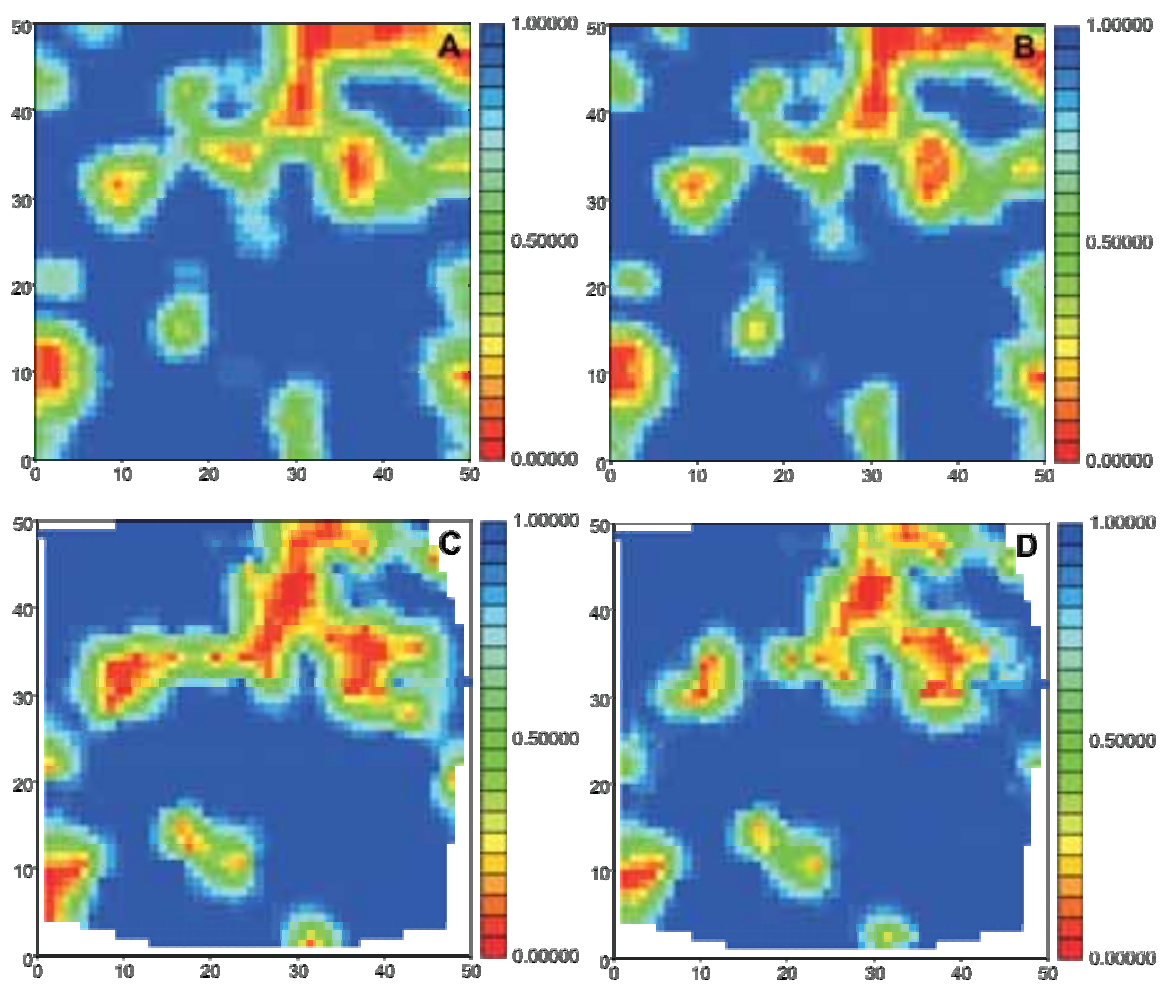

Figura 15. Mapas de probabilidades reais: peso constante (A) e peso variável (B) e mapas de probabilidade estimados pela PKI (C) e pela PKO (D) (teor de corte $=2,680$ ).

Considerando que os mapas de probabilidades estimados foram obtidos com uma amostra com 144 pontos, ou seja, 5,76\% do conjunto completo, se pode concluir que estes são uma razoável representação da realidade. Entre as duas aproximações comparadas, a krigagem ordinária sempre apresenta os melhores resultados que a técnica tradicional da krigagem da indicadora da mediana. As funções de distribuição acumulada condicionais derivadas da krigagem da indicadora da mediana são monotônicas crescentes em degraus, devido à transformação binária utilizada para obtenção da variável indicadora. Por outro lado, aquelas derivadas diretamente dos pesos da krigagem ordinária são mais contínuas e se correlacionam melhor às funções de distribuição acumulada reais.

\section{CONCLUSÕES}

Os resultados mostraram que é possível derivar probabilidades condicionais diretamente dos pesos da krigagem ordinária, com grandes vantagens em relação àquelas calculadas pela krigagem da indicadora da mediana. Na verdade, os mapas de probabilidade podem ser calculados no processo de estimativa através da krigagem ordinária, com grande economia de tempo.

\section{AGRADECIMENTOS}

$\mathrm{O}$ autor expressa os seus agradecimentos ao CNPq, através da concessão de bolsa de produtividade em pesquisa (Processo CNPq 303505/2007-9). O autor também agradece as sugestões feitas por dois revisores anônimos que melhoraram o manuscrito submetido.

\section{REFERÊNCIAS}

DEUTSCH, C. V.; JOURNEL, A. G. GSLIB: Geostatistical software library and user's guide. New York: Oxford University Press, 1992. 340 p.

DEUSTSCH, C. V.; JOURNEL, A. G. GSLIB: Geostatistical software library and user's guide. 2. ed. New York: Oxford University Press, 1998. 369 p.

HOHN, M. E. Geostatistics and petroleum geology. Boston: Kluwer Academic Publishers, 1999. 235 p.

JOURNEL, A. G.; RAO, S. E. Deriving conditional distributions from ordinary kriging. Palo Alto: Stanford Center for Reservoir Forecasting. 1996, 25 p. (Report 9). 
OLEA, R. Geostatistics for engineers and earth scientists. Boston: Kluwer Academic Publishers, 1999. 303 p.

YAMAMOTO, J. K. Comparação de métodos computacionais para avaliação de reservas: um estudo de caso no Depósito de Cobre de Chapada, GO. 1991. 1v. Tese (Doutorado) - Instituto de Geociências, Universidade de São Paulo, São Paulo, 1991.

YAMAMOTO, J. K. Convex_Hull - A Pascal program for determining the convex hull for planar sets. Computers \& Geosciences, Elmsford, v. 23, n. 7, p.725-738, 1997.

YAMAMOTO, J. K. An alternative measure of the reliability of ordinary kriging estimates. Mathematical Geology, New York, v. 32, no. 4, p. 489-509. 2000.

YAMAMOTO, J. K. Geoestatística prática usando o GeoVisual. São Paulo: Instituto de Geociências, Universidade de São Paulo. 2001. 53p. (Publicação interna do Laboratório de Informática Geológica).

YAMAMOTO, J. K. Correcting the smoothing effect of ordinary kriging estimates. Mathematical Geology, New York, v. 37, p. 69-94, 2005. 